\title{
Novel mesh-derived right ventricular free wall longitudinal strain analysis by intraoperative three-dimensional transoesophageal speckle-tracking echocardiography: a comparison with conventional parameters
}

\author{
Marius Keller ${ }^{1}$ (i) $\cdot$ Tobias Lang $^{2} \cdot$ Andreas Schilling $^{2} \cdot$ Martina Nowak-Machen $^{3} \cdot$ Peter Rosenberger $^{1} \cdot$ Harry Magunia $^{1}$
}

Received: 18 March 2019 / Accepted: 12 July 2019 / Published online: 18 July 2019

(c) Springer Nature B.V. 2019

\begin{abstract}
Longitudinal right ventricular (RV) function is substantial and might be reflected by free wall longitudinal strain (FWLS). Software solutions for FWLS analysis by two-dimensional (2D) and three-dimensional (3D) transesophageal echocardiography (TEE) are available, but data on validation are sparse. In this study, a novel method for FWLS analysis on 3D meshes ("mesh surface", MS-FWLS,) was tested for feasibility and compared to available parameters. 80 patients undergoing leftsided cardiac valve surgery with intraoperative TEE were included retrospectively. 2D-FWLS, 3D-derived (3Dd)-FWLS (assessed in optimized four-chamber views after volume analysis) and MS-FWLS were measured and compared to conventional parameters (3Dd-TAPSE, FAC and RVEF). The mean FWLS values did not differ significantly between methods $(-19.0 \pm 6.1 \%,-20.0 \pm 7.3 \%,-19.5 \pm 7.3 \%$ for 2D-, 3Dd- and MS-FWLS, respectively). No significant differences in the mean FWLS between patients with normal or increased pulmonary artery pressures as well as normal or reduced left ventricular ejection fraction were observed. Agreement was best between 3Dd- and MS-FWLS $(\mathrm{r}=0.89$, bias $=-1.0 \%$, $\mathrm{LOA} \pm 6.9 \%)$. Conventional echocardiographic parameters yielded poorer intermodality agreement. In patients with discrepant results between 2D- and 3Dd-FWLS, 3Dd-FWLS and MS-FWLS yielded similar results $(\mathrm{r}=0.82$, bias $=-0.3 \%$, LOA $\pm 8.6 \%$, while $2 \mathrm{D}-\mathrm{FWLS}$ and MS-FWLS did not. Intra- and interobserver variabilities of strain analyses were low. MS-FWLS might represent a promising method to overcome artefacts associated with $2 \mathrm{D}$ analysis. Its prognostic relevance needs to be investigated in prospective studies.
\end{abstract}

Keywords Right ventricle $\cdot$ Free wall strain $\cdot$ Transesopheageal echocardiography $\cdot$ Speckle-tracking $\cdot$ Three-dimensional

\section{Abbreviations}

2D-FWLS

2D free wall longitudinal strain

3Dd-FWLS 3D-derived free wall longitudinal strain

3Dd-TAPSE 3D-derived tricuspid annular plane systolic excursion

Marius Keller

marius.keller@med.uni-tuebingen.de

1 Department of Anaesthesiology and Intensive Care Medicine, University Hospital Tuebingen , Eberhard-KarlsUniversity, Hoppe-Seyler-Strasse 3, 72076 Tuebingen, Germany

2 Chair of Visual Computing, Department of Computer Science , Eberhard-Karls-University , Sand 14, 72076 Tuebingen, Germany

3 Institute of Anaesthesiology and Intensive Care Medicine, Klinikum Ingolstadt , Krumenauerstrasse 25, 85049 Ingolstadt, Germany
4CH FOUR-chamber view

ED end-diastole

ES End-systole

etCO $\mathrm{O}_{2} \quad$ End-tidal carbon dioxide

FAC Fractional area change

$\mathrm{FiO}_{2} \quad$ Fraction of inspired oxygen

FWd End-diastolic right ventricular free wall thickness

FWLS Free wall longitudinal strain

ICC Intraclass correlation coefficient

LV Left ventricle/left ventricular

LVEF Left ventricular ejection fraction

MAP Mean arterial pressure

$\mathrm{mmHg} \quad$ Millimetre of mercury

FWLS Mesh surface free wall longitudinal strain

ns Not (statistically) significant

NYHA New York Heart Association 


$\begin{array}{ll}\text { PAP }_{\text {sys }} & \text { Systolic pulmonary artery pressure } \\ \text { ROI } & \text { Region of interest } \\ \text { RV } & \text { Right ventricle/right ventricular } \\ \text { RVEDV } & \text { Right ventricular end-diastolic volume } \\ \text { RVEF } & \text { Right ventricular ejection fraction } \\ \text { RVESV } & \text { Right ventricular end-systolic volume } \\ \text { STE } & \text { Speckle-tracking echocardiography } \\ \text { STROBE } & \begin{array}{l}\text { Strengthening the Reporting of Observa- } \\ \text { tional Studies in Epidemiology }\end{array} \\ \text { TAPSE } & \text { Tricuspid annular plane systolic excursion } \\ \text { TEE } & \text { Transoesophageal echocardiography } \\ \text { TTE } & \text { Transthoracic echocardiography }\end{array}$

\section{Introduction}

Right ventricular (RV) dysfunction assessed by echocardiography is associated with a poor prognosis in the perioperative setting [1]. RV strain analysis recently became a promising method in addition to parameters like fractional area change (FAC) or ejection fraction (RVEF) [2]. Semiautomated speckle-tracking echocardiography (STE) has led to more robust and angle-independent results with better reproducibility compared to tissue Doppler imaging [3].

Free wall longitudinal strain (FWLS) is a major factor to right ventricular ejection, and its $2 \mathrm{D}$ assessment is recommended to establish a comprehensive understanding of right ventricular dysfunction [4]. Experimental 3D STE software packages have been used previously to measure right ventricular longitudinal strain on meshes derived from transthoracic echocardiography [5]. Only a few studies have focused on RV strain analysis using transoesophageal echocardiography (TEE); however, TEE represents the diagnostic tool of choice for the intraoperative assessment of cardiac function and has the ability to improve surgical decision making [6]. Data on intraoperative TEE strain analysis are limited and data on 3D mesh-based measurements using commercially available software packages are lacking [7].

In this study, available STE-based FWLS analysis methods from 2D images and 3D-derived optimized echocardiographic four-chamber views were compared to a novel parameter: the authors intended to measure FWLS on the surface of RV meshes derived from 3D TEE speckle-tracking volume analysis (MS-FWLS), potentially reflecting a valuable new tool to assess longitudinal RV function. This measurement is hypothesized to be feasible and validated by achieving comparable results as the aforementioned techniques. The three strain parameters were compared to conventional parameters of RV function. As a suitable study population, patients with mitral or aortic valve disease were chosen-reflecting intrinsically different pathologies regarding right heart function-in order to test the new technique on patients with a potential increase in preload (mainly mitral valve regurgitation) and afterload (mainly aortic stenosis).

\section{Materials and methods}

\section{Study type}

This is a feasibility cohort study using echocardiographic images available from a retrospectively recruited study population.

\section{Patients}

Consecutive patients who were scheduled for open left-sided cardiac valve surgery at the University Hospital Tuebingen between March 2014 and September 2017 were screened for study enrolment. Only patients over 18 years and with intraoperative TEE studies during haemodynamic stability with all images necessary for 2D and 3D strain analysis were included. The study was conducted in accordance with the Declaration of Helsinki and approved by the Ethics Committee of the University of Tuebingen (Trial Registration \# IRB 350/2015R).

\section{Anaesthesia management}

Anaesthetic management was performed by experienced cardiac anaesthesiologists. Anaesthesia induction was achieved intravenously by the administration of midazolam (0.1-0.15 mg/kg bw), sufentanil (0.3-0.5 $\mu \mathrm{g} / \mathrm{kg}$ bw) and rocuronium $(0.5-1 \mathrm{mg} / \mathrm{kg} \mathrm{bw})$. After endotracheal intubation, anaesthesia was maintained using sevoflurane. Physiological levels of etCO $\mathrm{C}_{2}$ and $\mathrm{SpO}_{2}$ were established, positive end-expiratory pressure values ranged between 3 and $6 \mathrm{cmH}_{2} \mathrm{O}$.

\section{Haemodynamics}

Patients received a volume-restrictive infusion regimen using an isotonic crystalloid solution ( $<15 \mathrm{ml} / \mathrm{kg} \mathrm{bw})$. To maintain a mean artery pressure (MAP) of $>65 \mathrm{mmHg}$, norepinephrine was the vasopressor of choice, administered by a titrated continuous infusion. Before cardiopulmonary bypass and after the establishment of haemodynamic stability, echocardiography was performed.

\section{Echocardiography protocol}

All echocardiographic data sets were acquired by experienced investigators. The institutional protocol has been previously described [8]. A commercially available 3D compatible TEE probe (Philips S7-2t Omni or Philips X7-2t 
Matrix, Philips Healthcare, Inc., Andover, MA, USA) was used to acquire a comprehensive TEE study of the left and right ventricle in accordance with current recommendations [9, 10]. 2D mid-oesophageal RV-focused 4-chamber view (4CH) was acquired with respect to optimal display of the whole RV free wall. FAC was assessed in 2D 4CH by averaging the measurements of two cardiac cycles. Wall motion abnormalities were not assessed. Multi-beat acquisition of 4 heart beats and a full projection of the right heart yielded a representative 3D loop for offline analysis. In comparison to 6-beat acquisition, multi-beat recordings of 4 heart beats yield frame rates of around $30 \mathrm{fps}$ and reduce the risk of stitching artefacts, creating optimal conditions for strain analysis. To guarantee comparable conditions regarding the haemodynamic state of the 2D and 3D data sets, only studies acquired in a near-simultaneous manner were regarded suitable, meaning: the TEE loops required for the analysis of all study measurements (2D 4CH first, 3D full volume directly afterwards) were recorded without a change in haemodynamic parameters or ventilator settings in between recordings.

\section{Two- and three-dimensional STE strain analysis}

All methods used in the study intended to measure peak systolic FWLS of the whole RV free wall endocardial contour from the lateral base of the tricuspid annulus to the RV apex. 2D- and MS-FWLS take shortening of the contour throughout the cardiac cycle into account. 3Dd-FWLS measures the shortening from end-diastole (R-wave-triggered) to endsystole (frame with the smallest mesh volume), potentially disregarding post-systolic strains. Strain is defined by the relative shortening from end-diastole to end-systole and is expressed as a percentage (\%). More negative strain values were regarded as higher strains, and less negative values were regarded as lower strains. Measurements were performed offline by two experienced investigators (MK, HM) and blinded to clinical data.

For the 2D-FWLS, we used an established STE software algorithm which contains a special mode to track the RV (2D CPA, Tomtec Imaging Systems GmbH, Unterschleissheim, Germany). The 2D-FWLS was measured in $4 \mathrm{CH}$ by placing multiple points along the endocardial border in a single frame. This region of interest (ROI) included the whole RV endocardium (Fig. 1a) and was then traced automatically throughout the cardiac cycle. Manual correction was obtained if necessary.

Using a comprehensive three-dimensional RV STEbased software package (4D RV-Function 2.0, Tomtec Imaging Systems GmbH, Unterschleissheim, Germany), strain analysis can be described as a side product of volume quantification. After the adjustment of several 2D views of the 3D echocardiographic data set (Fig. 1b), the software automatically detects the endocardial borders of the RV at end-diastole and displays the results in multiple views. Manual correction of the endocardial borders was performed if necessary, while simultaneously reviewing the tracking quality in the loop mode of the entire cardiac cycle. The resulting mesh-representing the endocardial filling volume of the $\mathrm{RV}$ - can be investigated from various angles during the whole analysis. While this complex and time-consuming quantification process mainly yields RV volumes and RVEF, the software also creates an optimized 3D-derived two-dimensional four-chamber view, which is displayed in the end-diastolic and end-systolic frames (Fig. 1b). The corresponding endocardial ROI represents a 3D-derived free wall longitudinal strain (3Dd-FWLS) with the possibility to correct the ROI manually in both views. Tricuspid annular plane systolic excursion (3Dd-TAPSE) was assessed in the same step.

\section{Novel 3D-derived mesh surface strain analysis}

The measurement of novel mesh surface free wall longitudinal strain (MS-FWLS) was performed on meshes derived from the 3D-STE RV analysis mentioned above. A second mesh was generated for each patient from the same 3D echo recording so that the MS-FWLS and 3Dd-FWLS analyses were not performed on the exact same data sets. The basal ROI insertion point was defined as the most lateral and basal pixel of the tricuspid annulus, while the most apical pixel served as the apical insertion point (Fig. 1c). After initial selection of the basal and apical mesh points those same points were used on all datasets. The shortest distance between the basal and the apical insertion point alongside the mesh surface was calculated using a custom-made software written in $\mathrm{C}++$ based on the Visualization Toolkit (Ver. 7.1.1, Kitware, Inc., Clifton Park, New York, USA).

\section{Analysis of reproducibility and practicability}

To assess the reproducibility of each strain method, 8 patients were randomly chosen from the study population. Blinded analysis by the same investigator yielded intraobserver variability, while blinded analysis by a second investigator yielded interobserver variability.

The time necessary for strain analysis was recorded from the beginning of the measurements until the display of the results.

\section{Statistical analysis}

Strain values are shown in percentages (\%). If samples were normally distributed according to the D'Agostino-Pearson test, the mean values \pm standard deviations and the $95 \%$ confidence interval of the mean (in square brackets) were 
a

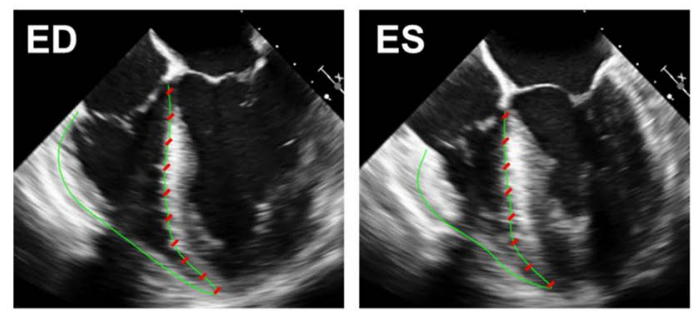

2D-FWLS

b
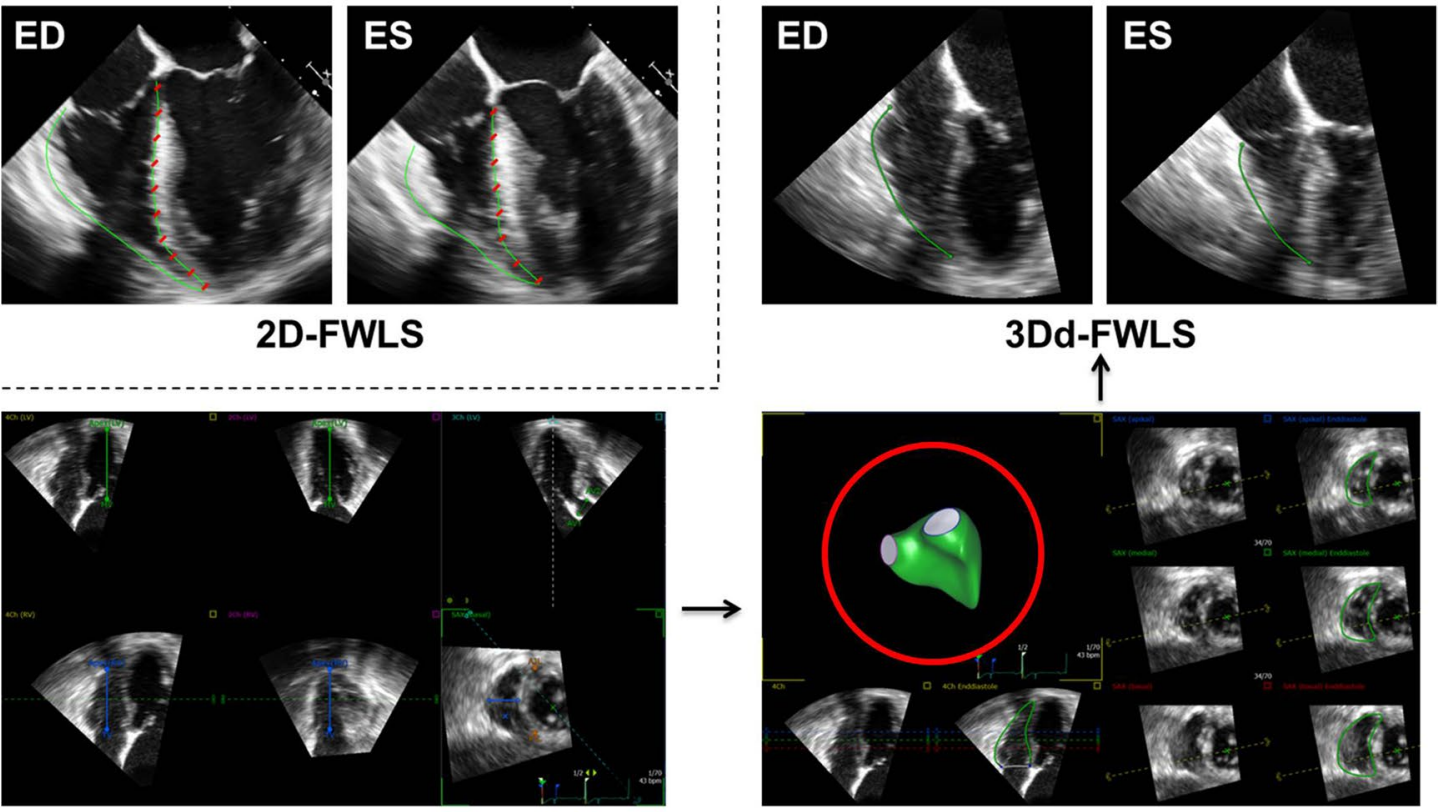

3Dd-FWLS

$\uparrow$
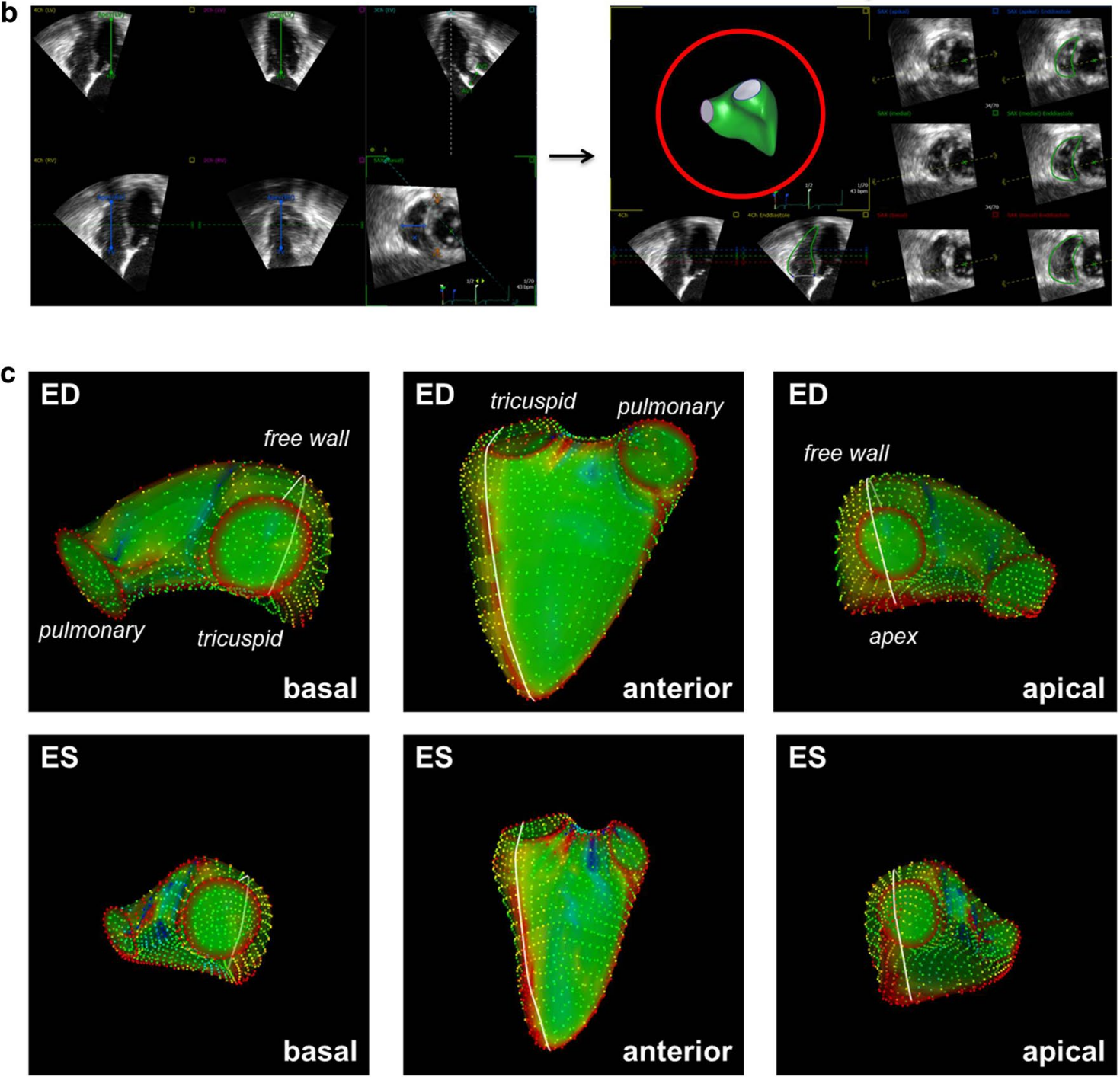

Fig. 1 a Free wall longitudinal strain (FWLS, green lines) analysis by two-dimensional (2D) speckle-tracking echocardiography (2D-FWLS) in four-chamber view $(4 \mathrm{CH})$. The septal wall was excluded for analysis (red lines). b Adjustment of planes for three-dimensional (3D) right ventricular volumetry (lower left), validation of endocardial border detection by speckle-tracking (lower right) and 3D-derived 2D

used to describe the sample. Ordinal data are displayed using mean with the 25th and 75th percentile in brackets. Sample distributions were illustrated using boxplot diagrams (line strain analysis (3Dd-FWLS) in an optimized 4CH (upper right). c Free wall longitudinal strain analysis (white line) on the endocardial surface of mesh data sets (red circle in $\mathbf{b}$ ) derived from three-dimensional speckle-tracking right ventricular volumetry (MS-FWLS). Frames are shown in end-diastole (ED) and end-systole (ES) in basal (left), anterior (center) and apical views (right) of the right ventricle

within box: median, box borders: 25 th and 75 th percentile, antennas: minimum to maximum). The different methods for strain measurement were compared using the paired 
Student's t-test with Welch's correction. The mean values of the subgroups were compared using the unpaired Student's t-test. The p-values were considered statistically significant as follows: moderately for $\mathrm{p}<0.05(*)$, medium for $\mathrm{p}<0.01(* *)$, and highly for $\mathrm{p}<0.001(* * *)$. In the case of normal distribution, Pearson's correlation coefficients were calculated; otherwise, Spearman's coefficients were used. The differences of proportions between two samples were tested using the Chi-squared test.

The Bland-Altman limits of agreement (LOA) were defined as the interval of bias \pm 1.96 -fold standard deviation containing $95 \%$ of the measurements. For the analysis of intraobserver and interobserver agreement, intraclass correlation coefficients were calculated.

Excel 2010 (Microsoft Corp., Redmond, WA, USA) and Prism 6 (GraphPad Software, Inc., La Jolla, CA, USA) were used for data documentation and statistical analysis. The presentation of data was performed in accordance with the STROBE statement [11].

\section{Results}

\section{Patient characteristics and echocardiographic image quality}

Of 455 screened patients for study inclusion, 92 patients had TEE studies according to the protocol that matched the inclusion criteria and were primarily enrolled (Fig. 2). $\mathrm{N}=12$ patients were excluded due insufficient 2D TEE image quality or artefacts, leaving 80 patients included for the final analysis (87\%). The 3D TEE acquisitions were rated suitable in all patients $(n=92,100 \%)$. The frame rates of the studies used for strain analysis were $50 \pm 1$ and
$28 \pm 5$ frames per second for $2 \mathrm{D}$ and $3 \mathrm{D}$ datasets, respectively $(\mathrm{p}<0.001)$. The mean heart rate during $2 \mathrm{D}$ and $3 \mathrm{D}$ acquisitions was $64 \pm 16$ beats per minute. Baseline data of the study population are shown in Table 1.

\section{Strain analysis}

Mean strain values for the whole study population $(\mathrm{n}=80)$ were $-19.0 \pm 6.1 \%[-20.4,-17.7],-20.0 \pm 7.3 \%$ [-21.6, $-18.4]$ and $-19.5 \pm 7.3 \%$ [-21.1, - 17.8] for 2D-FWLS, 3Dd-FWLS and MS-FWLS, respectively. The results did not differ significantly across methods. Boxplots, correlation diagrams and the Bland-Altman analyses are shown in Fig. 3. Agreement between 3Dd-FWLS and MS-FWLS was slightly better than for the comparison of 2D-FWLS versus 3Dd-FWLS and 2D-FWLS versus MS-FWLS. The linear regression and correlation analysis were highly significant $(\mathrm{p}<0.001)$ for all comparisons.

Table 2 shows the different FWLS results for the subdivision of patient groups according to the type of surgery, in case of increased pulmonary artery pressure (PAP) or decreased left ventricular ejection fraction (LVEF). No significant differences in FWLS between the groups were found in any of the applied methods.

\section{Conventional parameters}

Overall, 3Dd-TAPSE was $14 \pm 5 \mathrm{~mm}$, FAC was $40 \pm 10 \%$ and RVEF was $41 \pm 11 \%$. The correlation between FAC and RVEF was weak $(r=0.35, p=0.002)$. No significant differences between patients with normal or increased PAP as
Fig. 2 Flow chart of the study design and the inclusion of eligible patients. $2 D$ two-dimensional, $3 D$ three-dimensional, $C P B$ cardiopulmonary bypass, $T E E$ transoesophageal echocardiography

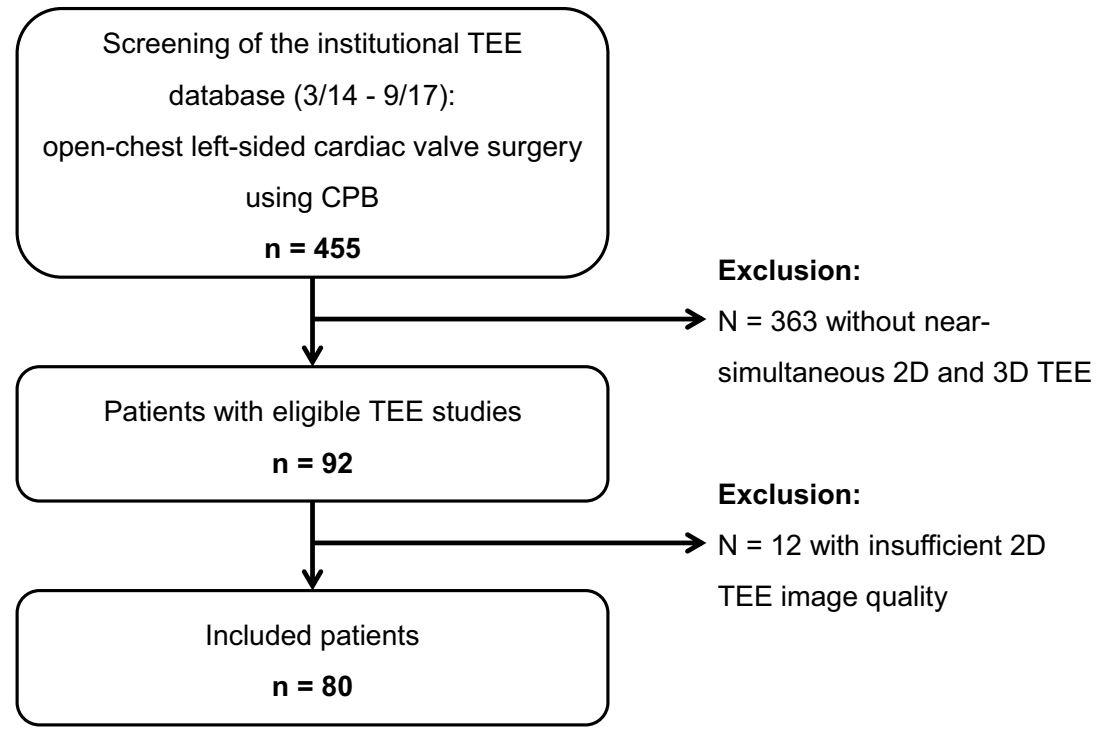


Table 1 Epidemiological, clinical and baseline characteristics of the study population

\begin{tabular}{|c|c|c|c|c|}
\hline & All patients & Aortic valve surgery & Mitral valve surgery & p-value \\
\hline Number, $\mathrm{n}$ & $80(100 \%)$ & $33(41 \%)$ & $47(59 \%)$ & 0.17 \\
\hline Male, $\mathrm{n}$ & $48(60 \%)$ & $21(64 \%)$ & $27(57 \%)$ & 0.86 \\
\hline Age (years) & $60 \pm 16$ & $59 \pm 17$ & $60 \pm 16$ & 0.79 \\
\hline NYHA class & II (II, III) & II (II, III) & II (II, III) & - \\
\hline \multicolumn{5}{|l|}{ Type of surgery } \\
\hline Replacement & $50(63 \%)$ & $32(97 \%)$ & $18(38 \%)$ & $<0.001 * * *$ \\
\hline Reconstruction & $30(37 \%)$ & $1(3 \%)$ & $29(62 \%)$ & $<0.001 * * *$ \\
\hline MAP, $\mathrm{mmHg}^{\mathrm{a}}$ & $80 \pm 16$ & $81 \pm 18$ & $79 \pm 15$ & 0.59 \\
\hline Reduced LVEF, $\mathrm{n}^{\mathrm{b}}$ & $20(25 \%)$ & $11(33 \%)$ & $9(19 \%)$ & 0.24 \\
\hline Increased $\mathrm{PAP}_{\mathrm{sys}}, \mathrm{n}^{\mathrm{c}}$ & $21(26 \%)$ & $4(12 \%)$ & $17(36 \%)$ & $0.03 *$ \\
\hline $\mathrm{FWd}, \mathrm{mm}$ & $8.8 \pm 2.1$ & $9.0 \pm 2.2$ & $8.7 \pm 2.1$ & 0.55 \\
\hline RVEDV, ml & $137 \pm 41$ & $130 \pm 35$ & $142 \pm 44$ & 0.20 \\
\hline RVESV, ml & $81 \pm 33$ & $75 \pm 30$ & $84 \pm 35$ & 0.23 \\
\hline
\end{tabular}

NYHA New York Heart Association, FWd end-diastolic right ventricular free wall thickness, $R V E D V$ right ventricular end-diastolic volume, $R V E S V$ right ventricular end-systolic volume (PAP and volumes derived from intraoperative TEE)

${ }^{a}$ MAP (mean artery pressure) during haemodynamic stability and TEE

${ }^{b}$ LVEF (left ventricular ejection fraction) considered reduced if $<50 \%$ preoperatively

${ }^{c} \mathrm{PAP}_{\text {sys }}$ (systolic pulmonary artery pressure) considered increased if $>30 \mathrm{mmHg}$ well as between patients with normal or reduced LVEF were observed for 3Dd-TAPSE, FAC or RVEF (Table 3).

Correlation coefficients for the comparison of RVEF with 2D-FWLS, 3Dd-FWLS and MS-FWLS were - 0.59, - 0.72 and -0.72 , respectively. For 3Dd-TAPSE, correlation coefficients were $-0.62,-0.69$ and -0.68 for the comparison with 2D-FWLS, 3Dd-FWLS and MS-FWLS, respectively. Correlation coefficients between FAC and 2D-FWLS, 3DdFWLS and MS-FWLS were $-0.40,-0.38$ and -0.37 respectively.

\section{Analysis of discrepancies between the 2D- and 3Dd-FWLS}

For the specific subanalysis of discrepancies between 2D-FWLS and 3Dd-FWLS measurements, patients with a difference between both methods below the 10th percentile $(-8.2 \%)$ and above the 90th percentile $(+6.0 \%)$ were considered separately $(n=16)$. In these patients, agreement between 3Dd-FWLS and MS-FWLS remained good $(\mathrm{r}=0.83, \mathrm{p}<0.001$, bias $=-0.3 \%, \mathrm{LOA} \pm 8.6 \%)$, while the comparison of 2D-FWLS and MS-FWLS revealed low agreement $(r=0.15, p=0.6$, bias $=1.5 \%, \mathrm{LOA} \pm 16.5 \%$; see Fig. 4).

For the same subanalysis, correlation coefficients $(r)$ were $-0.49(\mathrm{p}=0.05),-0.74(\mathrm{p}<0.001)$ and $-0.75(\mathrm{p}<0.001)$ for the comparison of RVEF with 2D-FWLS, 3Dd-FWLS and MS-FWLS, respectively. Correlation of FAC with 2D-FWLS, 3Dd-FWLS and MS-FWLS in these patients was weak with $r=-0.30(p=0.28), r=-0.20(p=0.47)$ and $\mathrm{r}=-0.21(\mathrm{p}=0.44)$, respectively.

\section{Reproducibility and practicability}

Intraobserver and interobserver agreement parameters are listed in Table 4. Overall, the analysis of MS-FWLS yielded the most reproducible results of all strain measurements applied in the study.

The 3Dd-FWLS and 2D-FWLS analysis took $161 \pm 37 \mathrm{~s}$ and $64 \pm 21 \mathrm{~s}$, respectively. The additional time necessary for the MS-FWLS analysis using our software was $5 \pm 1 \mathrm{~s}$.

\section{Discussion}

To our knowledge, this is the first study dedicated to the systematic comparison of 2D and 3D-derived right ventricular free wall longitudinal strain analysis in transoesophageal echocardiographic images. Furthermore, it is the first approach to measure MS-FWLS derived from meshes from 3D TEE speckle-tracking volume analysis.

Fig. 3 a Boxplot diagrams of free wall longitudinal strain (FWLS) measurements by two-dimensional (2D) FWLS, three-dimensionalderived (3Dd) FWLS and mesh surface (MS) FWLS analysis. b Linear regression diagrams and correlation coefficients (r) are on the left, and Bland-Altman analyses are on the right for the comparisons of 2D-FWLS, 3Dd-FWLS and MS-FWLS analysis. LOA 95\% limits of agreement, $n s$ difference not statistically significant 
a
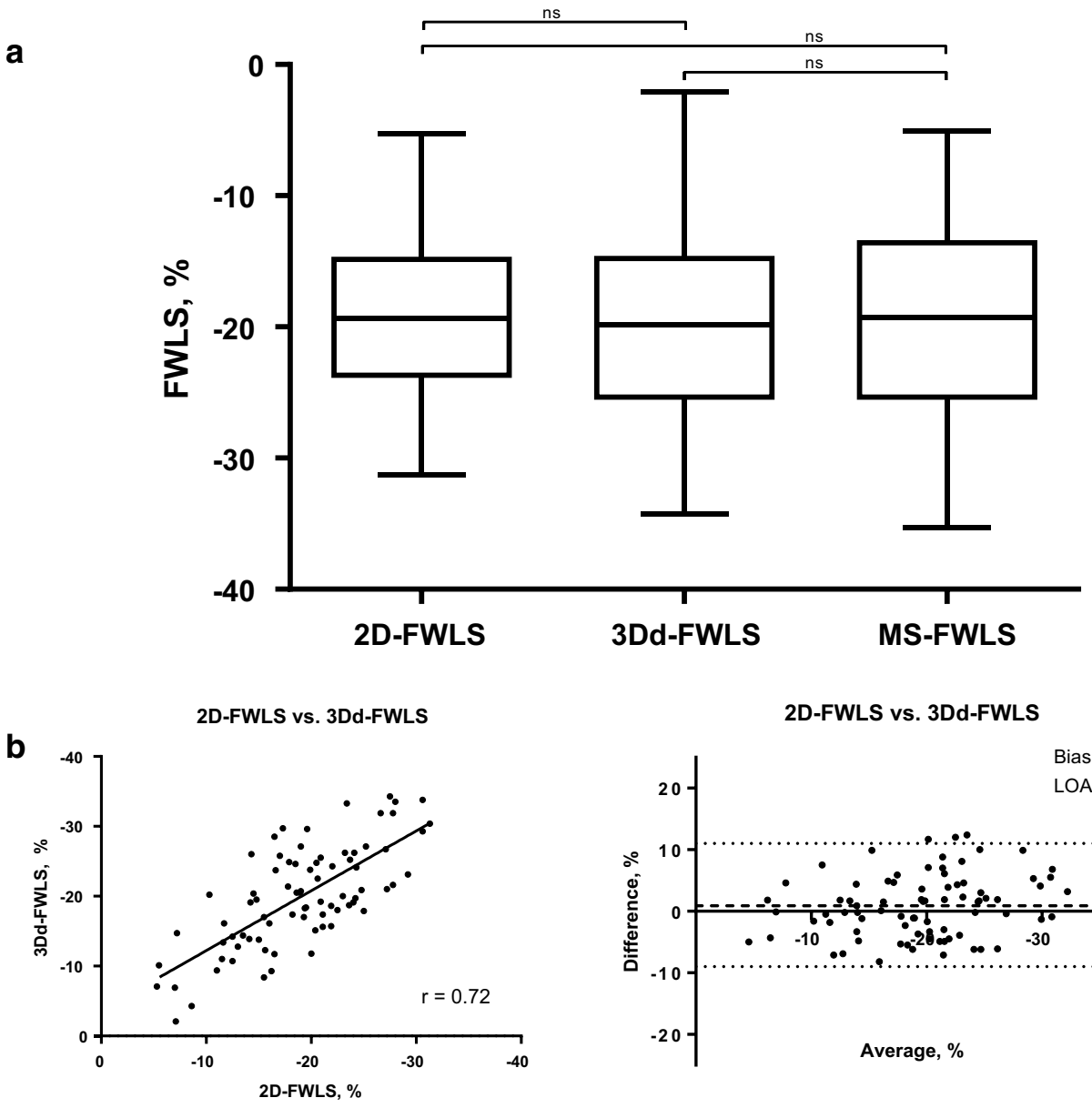

2D-FWLS vs. 3Dd-FWLS

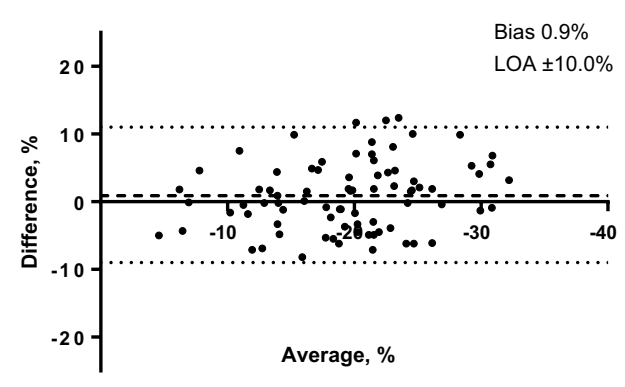

2D-FWLS vs. MS-FWLS

2D-FWLS vs. MS-FWLS
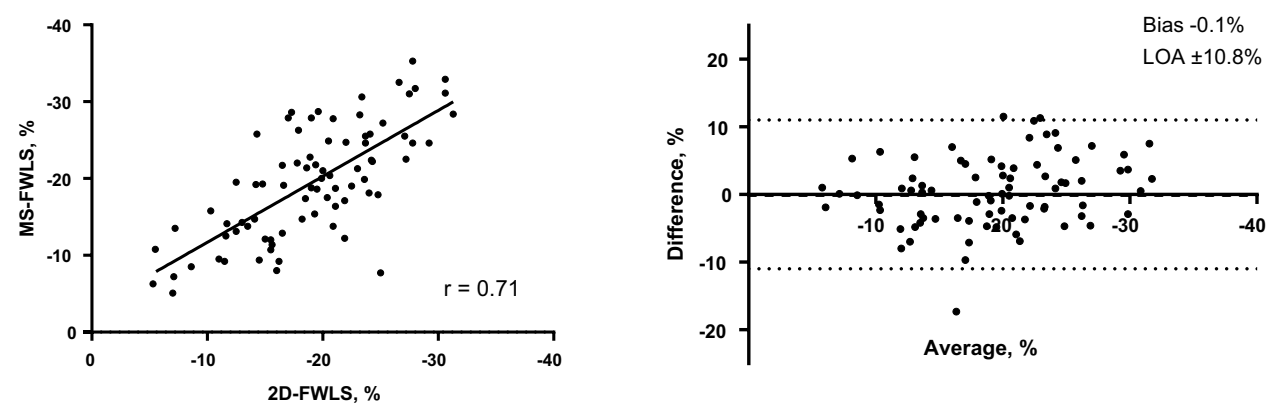

3Dd-FWLS vs. MS-FWLS

3Dd-FWLS vs. MS-FWLS
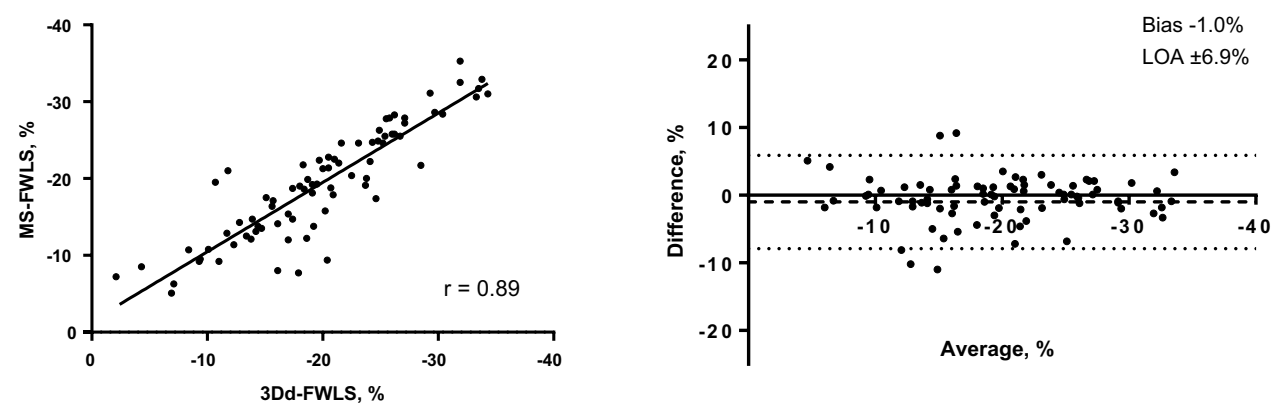
Table 2 Results of free wall longitudinal strain (FWLS) measurements by two-dimensional (2D-FWLS), three-dimensional-derived (3Dd-FWLS) and mesh surface (MS-FWLS) analysis subdivided by the type of surgery, pulmonary artery pressure and left-ventricular systolic function

\begin{tabular}{|c|c|c|c|}
\hline & Aortic valve patients $(\mathrm{n}=33)$ & $\begin{array}{l}\text { Mitral valve patients } \\
(\mathrm{n}=47)\end{array}$ & p-value \\
\hline 2D-FWLS & $\begin{array}{l}-19.5 \pm 6.4 \% \\
{[-21.8,-17.2]}\end{array}$ & $\begin{array}{l}-18.7 \pm 6.0 \% \\
{[20.5,-20.0]}\end{array}$ & 0.59 \\
\hline 3Dd-FWLS & $\begin{array}{l}-19.7 \pm 8.0 \% \\
{[-22.6,-16.9]}\end{array}$ & $\begin{array}{l}-20.1 \pm 6.7 \% \\
{[-22.1,-18.2]}\end{array}$ & 0.81 \\
\hline \multirow[t]{2}{*}{ MS-FWLS } & $\begin{array}{l}-19.6 \pm 8.0 \% \\
{[-22.4,-16.8]}\end{array}$ & $\begin{array}{l}-19.3 \pm 6.9 \% \\
{[-21.4,-17,3]}\end{array}$ & 0.88 \\
\hline & $\begin{array}{l}\text { Normal PAP } \\
(\mathrm{n}=59)\end{array}$ & $\begin{array}{l}\text { Increased PAP } \\
(n=21)\end{array}$ & p-value \\
\hline 2D-FWLS & $\begin{array}{l}-20.4 \pm 7.2 \% \\
{[-22.2,-18.5]}\end{array}$ & $\begin{array}{l}-18.4 \pm 6.7 \% \\
{[-21.1,-15.0]}\end{array}$ & 0.19 \\
\hline 3Dd-FWLS & $\begin{array}{l}-19.4 \pm 6.0 \% \\
{[-20.9,-17.9]}\end{array}$ & $\begin{array}{l}-18.9 \pm 7.6 \% \\
{[-22.3,-15.4]}\end{array}$ & 0.77 \\
\hline \multirow[t]{2}{*}{ MS-FWLS } & $\begin{array}{l}-19.6 \pm 7.5 \% \\
{[-21.5,-17.6]}\end{array}$ & $\begin{array}{l}-19.2 \pm 7.1 \% \\
{[-22.4,-15.9]}\end{array}$ & 0.83 \\
\hline & $\begin{array}{l}\text { Normal LVEF } \\
(n=60)\end{array}$ & $\begin{array}{l}\text { Reduced LVEF } \\
(\mathrm{n}=20)\end{array}$ & p-value \\
\hline 2D-FWLS & $\begin{array}{l}-19.8 \pm 6.2 \% \\
{[-21.4,-18.2]}\end{array}$ & $\begin{array}{l}-16.8 \pm 5.4 \% \\
{[-19.3,-14.3]}\end{array}$ & 0.11 \\
\hline 3Dd-FWLS & $\begin{array}{l}-20.5 \pm 7.3 \% \\
{[-22.4,-18.6]}\end{array}$ & $\begin{array}{l}-18.3 \pm 7.0 \% \\
{[-21.6,-15.0]}\end{array}$ & 0.37 \\
\hline MS-FWLS & $\begin{array}{l}-19.9 \pm 7.4 \% \\
{[-21.8,-18.1]}\end{array}$ & $\begin{array}{l}-18.1 \pm 7.0 \% \\
{[-21.4,-14.8]}\end{array}$ & 0.45 \\
\hline
\end{tabular}

$\mathrm{PAP}_{\text {sys }}$ (systolic pulmonary artery pressure) considered increased if $>30 \mathrm{mmHg}$ intraoperatively, LVEF (left ventricular ejection fraction) considered reduced if $<50 \%$ preoperatively

Table 3 Results of three-dimensional-derived tricuspid annular plane systolic excursion (3Dd-TAPSE), fractional area change (FAC) and right ventricular ejection fraction (RVEF) subdivided by pulmonary artery pressure and left ventricular systolic function

\begin{tabular}{llll}
\hline & $\begin{array}{l}\text { Normal PAP } \\
(\mathrm{n}=59)\end{array}$ & $\begin{array}{l}\text { Increased PAP } \\
(\mathrm{n}=21)\end{array}$ & p-value \\
\hline 3Dd-TAPSE & $15 \pm 5 \mathrm{~mm}$ & $14 \pm 5 \mathrm{~mm}$ & 0.53 \\
FAC & $40 \pm 10 \%$ & $38 \pm 12 \%$ & 0.40 \\
RVEF & $42 \pm 11 \%$ & $39 \pm 12 \%$ & 0.28 \\
\hline & Normal LVEF & Reduced LVEF & (n=20) \\
\hline 3Dd-TAPSE & $(\mathrm{n}=60)$ & $14 \pm 5 \mathrm{~mm}$ & 0.44 \\
FAC & $15 \pm 5 \mathrm{~mm}$ & $39 \pm 9 \%$ & 0.78 \\
RVEF & $40 \pm 11 \%$ & $38 \pm 11 \%$ & 0.19 \\
\hline
\end{tabular}

$\mathrm{PAP}_{\text {sys }}$ (systolic pulmonary artery pressure) considered increased if $>30 \mathrm{mmHg}$ intraoperatively, LVEF (left ventricular ejection fraction) considered reduced if $<50 \%$ preoperatively

The 2D-FWLS and 3Dd-FWLS measurements yielded fairly comparable results, yet discrepancies occurred in some patients. When considering patients with high discrepancies between 2D-FWLS and 3Dd-FWLS alone, MS-FWLS analysis yielded results that correlated better with 3Dd-FWLS than with 2D-FWLS.
No differences in the FWLS mean values were found between patients with normal PAP compared to patients with increased PAP. The authors attribute this to the fact that the criteria of haemodynamic stability were the same in both subgroups using a standardized anaesthetic management strategy. This might result in a "normalization" of 
( $\triangle 2 D / 3 D d)$ 3Dd-FWLS vs. MS-FWLS

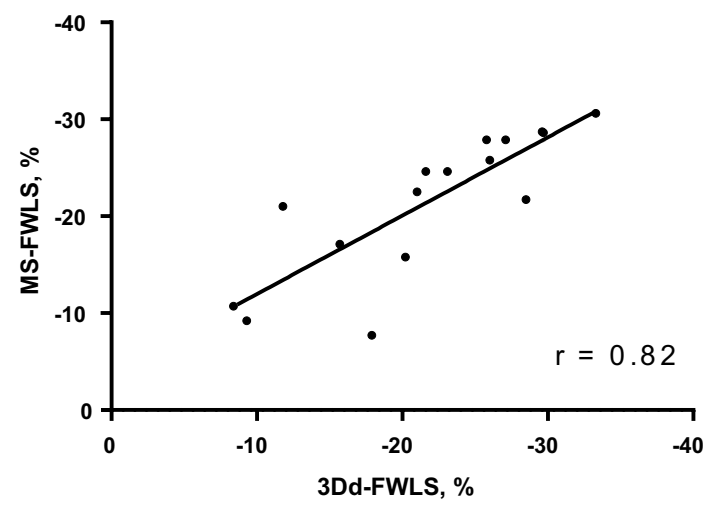

( $\triangle 2 D / 3 D d)$ 2D-FWLS vs. MS-FWLS

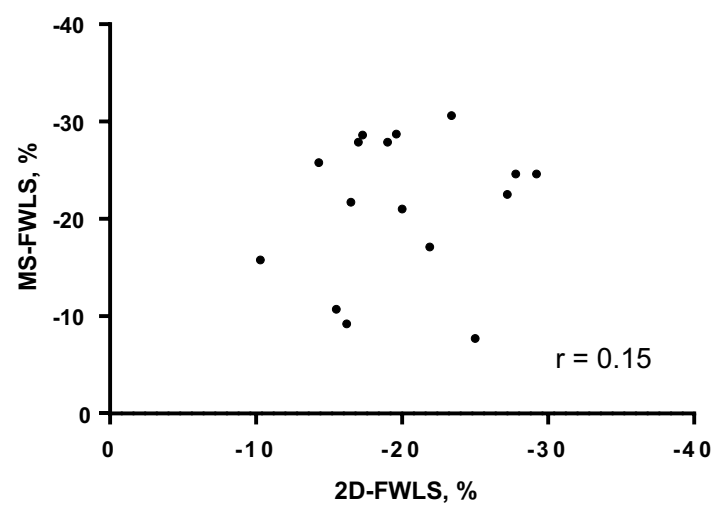

( $\triangle 2 D / 3 D d)$ 3Dd-FWLS vs. MS-FWLS

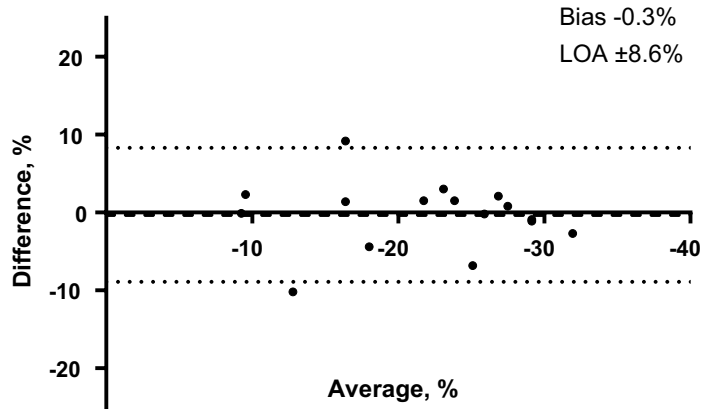

( $\triangle 2 \mathrm{D} / 3 \mathrm{Dd})$ 2D-FWLS vs. MS-FWLS

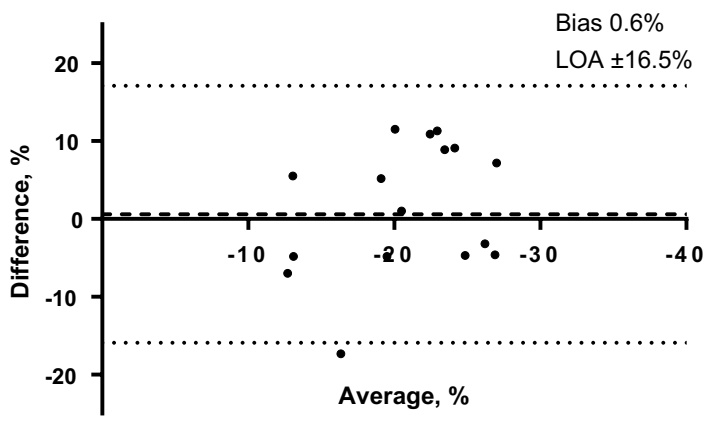

Fig. 4 Analysis of the discrepant free wall longitudinal strain (FWLS) results between 2D-FWLS and 3Dd-FWLS $(\Delta 2 \mathrm{D} / 3 \mathrm{Dd}, \mathrm{n}=16)$. Linear regression/plot diagrams and correlation coefficients (r) are on the left, Bland-Altman analysis is on the right. LOA $95 \%$ limits of agreement

load-dependent right ventricular myocardial deformation. Similar results were observed for the comparison of patients with normal or depressed systolic LV function.

The conventional parameters did not generate different results for patients with or without increased PAP or patients with normal or reduced LVEF, respectively. The 3D parameters (RVEF and 3Dd-TAPSE) showed better agreement with the strain results than 2D FAC. Surprisingly, while strain analysis as well as RVEF and TAPSE all suggested

Table 4 Intraobserver and interobserver agreement analysis

\begin{tabular}{|c|c|c|c|c|}
\hline \multirow[t]{2}{*}{ Method } & \multicolumn{2}{|c|}{ Intraobserver $(\mathrm{n}=8)$} & \multicolumn{2}{|c|}{$\begin{array}{l}\text { Interobserver } \\
(\mathrm{n}=8)\end{array}$} \\
\hline & $\mathrm{r}$ & ICC & $\mathrm{r}$ & ICC \\
\hline 2D-FWLS & 0.98 & 0.940 & 0.89 & 0.878 \\
\hline 3Dd-FWLS & 0.96 & 0.945 & 0.90 & 0.907 \\
\hline MS-FWLS & 0.97 & 0.969 & 0.98 & 0.972 \\
\hline
\end{tabular}

$R$ correlation coefficient, $I C C$ intraclass correlation coefficient decreased mean RV function in the investigated study population, mean FAC - the 2D counterpart of RVEF - was within the normal range of $>35 \%$, potentially reflecting a limitation of the method regarding its sensitivity during intraoperative TEE.

Intraobserver and interobserver analysis revealed good overall reproducibility with MS-FWLS being the most robust parameter. 3D strain analysis required approximately three times more time than 2D measurements.

This study demonstrates that 3D-based STE analysis of the FWLS is feasible on intraoperatively acquired TEE images. However, both image acquisition and the analysis itself were more sophisticated than the measurement of the same parameter in corresponding 2D images. The methods yielded divergent results in a fair amount of patients, leaving a few unanswered questions. It remains unclear which method is connected more closely to the measurement of the true FWLS.

It seems evident that $3 \mathrm{D}$ analysis is associated with some advantages compared to $2 \mathrm{D}$ analysis for the assessment of 
the FWLS. A specific potential error associated with 2D strain analysis is out-of-plane movement. Out-of-plane movement is present when the most longitudinal diameter of the RV free wall - ranging from the lateral tricuspid annulus to the apex - leaves the focused 2D plane over time; for example by shortening in a different axis than the $2 \mathrm{D}$ plane is oriented or by twisting out of plane [12]. A second artefact hindering the correct assessment of free wall strain is apical foreshortening; if the 2D plane does not strictly "cut through" the RV apex, a shortened version of the anticipated plane develops, especially during long-axis systolic shortening. In these foreshortened views, the results of the strain analysis can be altered [13].

These confounders can be avoided by using 3D fullvolume acquisition, where all structures necessary for FWLS analysis are visible throughout the cardiac cycle. However, a high temporal resolution is necessary for valid STE-based strain analysis. The 3D acquisition is associated with significantly lower frame rates compared to $2 \mathrm{D}$ TTE, and the risk for potential underestimation of peak strain values is higher [14]. To achieve sufficient frame rates, multi-beat acquisition became essential for threedimensional echocardiographic recordings [15]. Yet, in our study, both 3D parameters of FWLS yielded higher strains than 2D analysis, despite the higher frame rates of the 2D images. This phenomenon might underline the greater risk of underestimation of free wall strains associated with 2D artefacts compared to lower 3D frame rates. During tachycardia, the risk of strain underestimation due to low frame rates might play a bigger role.

Longitudinal function assessed by strain analysis essentially contributes to both pressure generation and volume ejection and thus reflects a sensitive parameter in various right heart pathologies $[16,17]$. One of the most established parameters reflecting longitudinal RV function is TAPSE. Serving as a well-validated, easy-to-assess and prognostically significant tool for TTE, TAPSE comes with a few disadvantages regarding its applicability on TEE. By definition, TAPSE is measured in TTE apical $4 \mathrm{CH}$ images via M-MODE [4]. Deep transgastric RV inflow images come closest as a surrogate TEE plane to measure TAPSE, yet the display of the most longitudinal parameter of the RV can become problematic. Additionally, Korschin et al. recently reported a feasibility of transgastric RV inflow TAPSE of only 36\% after sternotomy [18]. Approaches avoiding M-Mode measurements might improve the feasibility of TEE 2D TAPSE but lack comprehensive validation [19]. In this investigation, TAPSE was therefore only measured in an optimized $4 \mathrm{CH}$ derived from $3 \mathrm{D}$ volume segmentation and not in 2D images.

A clear advantage of TAPSE, however, is the consideration of the free wall of the RV alone, even if mainly the basal free wall segment is considered [2]. An inclusion of the septal wall into the analysis of the RV function parameters is problematic due to its enormous dependency on the left ventricle. In this context, Garcia-Martin et al. unsurprisingly found the RV global longitudinal strain to be superior to the FWLS regarding the prediction of heart failure in patients with left ventricular dysfunction [20]. Confirming these results, a decrease in FWLS was not significantly associated with impaired LV function under general anaesthesia in our study. As impaired left ventricular function could act as a confounder regarding the assessment of RV function including the RV septal wall, Smith et al. investigated RV free wall strain using a dedicated STE-based software algorithm on 3D TTE images of healthy controls and patients with pulmonary hypertension [21]. Interestingly, strain parameters remained independent predictors of mortality in the patient cohort, while both RVEF and TAPSE were not significantly different between survivors and nonsurvivors.

This leads to the conclusion that the assessment of RV free wall strain provides additional information on right ventricular function compared to standard parameters. While being more load-dependent than STE-based strain analysis [22], RVEF alone only reflects the current output of the RV. The assessment of regional RVEF by $3 \mathrm{D}$ echocardiography has recently been proven to be feasible and is a potential tool to detect incremental or disease-specific alterations of RV systolic function beyond the implications of global RVEF [23]. Longitudinal RV strain might unmask early RV dysfunction, while RVEF is still preserved [24, 25]. Studies have emphasized the correlation between the RV longitudinal strain parameters and RVEF derived by either echocardiography or cardiac magnetic resonance imaging $[17,26]$. However, whether an agreement between the two methods is meaningful in this context - especially during right ventricular disease-remains questionable. In particular, the correlation between FWLS and regional RVEF in the setting of various RV pathologies needs to be investigated.

The authors' idea in this study was to measure FWLS on three-dimensional endocardial mesh models of the RV obtained by 3D-STE TEE. While a mesh is generated to assess RV volumes and RVEF, it seemed consistent to measure FWLS on its surface. Thus, the systolic shortening of the free wall can be quantified along the actual longitudinal $\mathrm{RV}$ axis in three-dimensional space, regardless of any right ventricular temporal or spatial motion. Additionally, the measurements appear independent of changes in RV geometry-such as excessive dilation or complex malformations as in Tetralogy of Fallot-potentially representing a promising approach to assess longitudinal RV function in those patients. After development of the software algorithm, the analysis was feasible in all patients included in the study and yielded interesting results. The prognostic relevance of this 
novel method for FWLS analysis needs to be investigated in further studies, especially regarding its value on intraoperative TEE images.

\section{Limitations}

Interstudy variability due to changes in preload or afterload between 2 and 3D image acquisition is possible, even though the images were recorded almost simultaneously. The strain analysis was performed only in patients under general anaesthesia and standardized conditions regarding haemodynamic and respiratory parameters, which might limit the transferability to awake and spontaneously breathing patients. No data regarding prognosis were assessed.

\section{Conclusion}

Right ventricular free wall longitudinal strain analysis on the surface of the endocardial mesh models generated by intraoperative three-dimensional transoesophageal speckletracking echocardiography is feasible and shows good comparability to the commercially available FWLS measurements. Prospective trials are necessary to evaluate whether this novel method of right ventricular function assessment adds prognostic significance compared to conventional parameters.

Acknowledgements The authors thank all employees of the cardiothoracic and cardiac anaesthesia units at University Hospital Tuebingen for supporting image acquisition.

Funding This work was supported by a grant from the Deutsche Forschungsgemeinschaft (DFG): Grant DFG-INST 2388/71-1 FUGG.

\section{Compliance with ethical standards}

Conflict of interest All authors (Marius Keller, Tobias Lang, Andreas Schilling, Martina Nowak-Machen, Peter Rosenberger and Harry Magunia) declare that they have no conflicts of interest.

\section{References}

1. Ternacle J, Berry M, Cognet T, Kloeckner M et al (2013) Prognostic value of right ventricular two-dimensional global strain in patients referred for cardiac surgery. J Am Soc Echocardiogr. 267:721-726. https://doi.org/10.1016/j.echo.2013.03.021

2. Longobardo L, Suma V, Jain R, Carerj S et al (2017) Role of two-dimensional speckle-tracking echocardiography strain in the assessment of right ventricular systolic function and comparison with conventional parameters. J Am Soc Echocardiogr 3010(937946):e6. https://doi.org/10.1016/j.echo.2017.06.016

3. Geyer H, Caracciolo G, Abe H, Wilansky S et al (2010) Assessment of myocardial mechanics using speckle tracking echocardiography: fundamentals and clinical applications. J Am Soc Echocardiogr 234:351-369. https://doi.org/10.1016/j. echo.2010.02.015

4. Lang RM, Badano LP, Mor-Avi V, Afilalo J et al (2015) Recommendations for cardiac chamber quantification by echocardiography in adults: an update from the American Society of Echocardiography and the European Association of Cardiovascular Imaging. J Am Soc Echocardiogr 281(1-39):e14. https://doi.org/10.1016/j. echo.2014.10.003

5. Ishizu T, Seo Y, Atsumi A, Tanaka YO et al (2017) Global and regional right ventricular function assessed by novel three-dimensional speckle-tracking echocardiography. J Am Soc Echocardiogr 3012:1203-1213. https://doi.org/10.1016/j.echo.2017.08.007

6. Eltzschig HK, Rosenberger P, Loffler M, Fox JA et al (2008) Impact of intraoperative transesophageal echocardiography on surgical decisions in 12,566 patients undergoing cardiac surgery. Ann Thorac Surg 853:845-852. https://doi.org/10.1016/j.athor acsur.2007.11.015

7. Magunia H, Dietrich C, Langer HF, Schibilsky D et al (2018) $3 \mathrm{D}$ echocardiography derived right ventricular function is associated with right ventricular failure and mid-term survival after left ventricular assist device implantation. Int $\mathrm{J}$ Cardiol. https://doi.org/10.1016/j.ijcard.2018.06.02610.1016/j. ijcard.2018.06.026.

8. Magunia H, Schmid E, Hilberath JN, Haberle L et al (2017) 2D Echocardiographic evaluation of right ventricular function correlates with 3D volumetric models in cardiac surgery patients. $\mathbf{J}$ Cardiothorac Vasc Anesth 312:595-601. https://doi.org/10.1053/j. jvca.2016.11.020

9. Lang RM, Badano LP, Tsang W, Adams DH et al (2012) EAE/ ASE recommendations for image acquisition and display using three-dimensional echocardiography. J Am Soc Echocardiogr 251:3-46. https://doi.org/10.1016/j.echo.2011.11.010

10. Hahn RT, Abraham T, Adams MS, Bruce CJ et al (2013) Guidelines for performing a comprehensive transesophageal echocardiographic examination: recommendations from the American Society of Echocardiography and the Society of Cardiovascular Anesthesiologists. J Am Soc Echocardiogr 269:921-964. https:// doi.org/10.1016/j.echo.2013.07.009

11. von Elm E, Altman DG, Egger M, Pocock SJ et al (2007) The strengthening the reporting of observational studies in epidemiology (STROBE) statement: guidelines for reporting observational studies. Lancet 3709596:1453-1457. https://doi.org/10.1016/ s0140-6736(07)61602-x

12. Wu VC, Takeuchi M, Otani K, Haruki N et al (2013) Effect of through-plane and twisting motion on left ventricular strain calculation: direct comparison between two-dimensional and three-dimensional speckle-tracking echocardiography. J Am Soc Echocardiogr 2611:1274-1281.e4. https://doi.org/10.1016/j. echo.2013.07.006

13. Jacobs LD, Salgo IS, Goonewardena S, Weinert L et al (2006) Rapid online quantification of left ventricular volume from realtime three-dimensional echocardiographic data. Eur Heart $\mathbf{J}$ 274:460-468. https://doi.org/10.1093/eurheartj/ehi666

14. Negishi K, Negishi T, Agler DA, Plana JC et al (2012) Role of temporal resolution in selection of the appropriate strain technique for evaluation of subclinical myocardial dysfunction. Echocardiography 293:334-339. https://doi.org/10.111 1/j.1540-8175.2011.01586.x

15. Yodwut C, Weinert L, Klas B, Lang RM et al (2012) Effects of frame rate on three-dimensional speckle-tracking-based measurements of myocardial deformation. J Am Soc Echocardiogr 259:978-985. https://doi.org/10.1016/j.echo.2012.06.001

16. Lakatos B, Tósér Z, Tokodi M, Doronina A et al (2017) Quantification of the relative contribution of the different right ventricular wall motion components to right ventricular ejection fraction: 
the revision method. Cardiovasc Ultrasound 15:8. https://doi. org/10.1186/s12947-017-0100-0

17. Lu KJ, Chen JX, Profitis K, Kearney LG et al (2015) Right ventricular global longitudinal strain is an independent predictor of right ventricular function: a multimodality study of cardiac magnetic resonance imaging, real time three-dimensional echocardiography and speckle tracking echocardiography. Echocardiography 326:966-974. https://doi.org/10.1111/echo.12783

18. Korshin A, Grønlykke L, Nilsson JC, Møller-Sørensen H et al (2018) The feasibility of tricuspid annular plane systolic excursion performed by transesophageal echocardiography throughout heart surgery and its interchangeability with transthoracic echocardiography. Int J Cardiovasc Imaging 347:1017-1028. https://doi. org/10.1007/s10554-018-1306-4

19. Morita Y, Nomoto K, Fischer GW (2016) Modified tricuspid annular plane systolic excursion using transesophageal echocardiography for assessment of right ventricular function. J Cardiothorac Vasc Anesth. 301:122-126. https://doi.org/10.1053/j. jvca.2015.07.024

20. Garcia-Martin A, Moya-Mur JL, Carbonell-San Roman SA, Garcia-Lledo A et al (2016) Four chamber right ventricular longitudinal strain versus right free wall longitudinal strain. Prognostic value in patients with left heart disease. Cardiol J 232:189-194. https://doi.org/10.5603/CJ.a2015.0079

21. Smith BCF, Dobson G, Dawson D, Charalampopoulos A et al (2014) Three-dimensional speckle tracking of the right ventricle: toward optimal quantification of right ventricular dysfunction in pulmonary hypertension. J Am Coll Cardiol 641:41-51. https:// doi.org/10.1016/j.jacc.2014.01.084
22. Kossaify A (2015) Echocardiographic assessment of the right ventricle, from the conventional approach to speckle tracking and three-dimensional imaging, and insights into the "right way" to explore the forgotten chamber. Clin Med Insights Cardiol 9:6575. https://doi.org/10.4137/CMC.S27462

23. Nowak-Machen M, Lang T, Schilling A, Mockenhaupt L et al (2019) Regional Right Ventricular Volume and Function Analysis Using Intraoperative 3-Dimensional Echocardiography-Derived Mesh Models. J Cardiothorac Vasc Anesth 336:1527-1532. https ://doi.org/10.1053/j.jvca.2019.02.011

24. Luo L, Zhu J, Chen J, Gao L et al (2016) Study of right ventricular function with preserved left ejection fraction by three-dimensional speckle tracking in uremic patients undergoing peritoneal dialysis. Int J Clin Exp Med 96:11113-11124

25. Greiner S, Heimisch M, Aurich M, Hess JA et al (2014) Multiplane two-dimensional strain echocardiography for segmental analysis of right ventricular mechanics: new-RV study. Clin Res Cardiol 10310:817-824. https://doi.org/10.1007/s0039 2-014-0723-1

26. Focardi M, Cameli M, Carbone SF, Massoni A et al (2015) Traditional and innovative echocardiographic parameters for the analysis of right ventricular performance in comparison with cardiac magnetic resonance. Eur Heart J Cardiovasc Imaging 161:47-52. https://doi.org/10.1093/ehjci/jeu156

Publisher's Note Springer Nature remains neutral with regard to jurisdictional claims in published maps and institutional affiliations. 